\title{
HYBRID METHOD FOR IMAGE CLASSIFICATION
}

\author{
Haseela H A \\ Department of Computer Science, University of Kerala
}

Article DOI: https://doi.org/10.36713/epra9564

DOI No: 10.36713/epra9564

\section{INTRODUCTION}

The work discuss regarding the performance ensemble based algorithms for image classification. Kather dataset were trained by, machine learning techniques such as KNearestNeighbour and deep learning algorithms by Xception models.

\section{RELATED WORK}

E.F.Ohata et.al(2021)[1] A novel transfer learning approach for the classification of histological images of colorectal cancer. The automatic identification of eight categories is proposed . tissues discovered during a CRC histological examination The structures of CNNs to extract characteristics are discussed in this study. DenseNet169 with SVM was the one that got the greatest results (RBF). This method is capable of discriminating between tissues found in evaluation of the histopathology of a CRC.

Cascianelli et.al [2] proposed dimensionality reduction strategies for CNN based classification of histopathological Images. In this research paper, dimensionality reduction strategies for CNN-based features used in computer-assisted histopathological image categorization. Test two well-known methodologies (Principal Component Analysis and Gaussian Random Projection) and suggest a novel reduction strategy based on the cross-correlation between feature vector components. The results show that CNN-based characteristics can be reduced by a large factor with just a minor loss of accuracy when compared to the original values. In this paper, VGG-F, VGG-S, and VGG-VD-16 networks are employed, as well as feature selection methods such as Gaussian Random Projection, and Principal Component Analysis, and finally classification using a KNN classifier.

Wang et.al [3] proposed a novel BCNN-based technique for histopathological image classification that first decomposes histopathological pictures into hematoxylin and eosin stain components before applying BCNN to fuse and improve feature representation.

Pham [4] proposed a method in which Autoencoding has long been known in deep learning as a valuable approach for collecting picture features from several layers and then configuring them for classification by deep neural networks. The time required to train a large number of artificial neurons is a practical barrier to autoencoder implementation.This research examines the impacts of texture scaling in the histology of colorectal cancer, which can lead to a large reduction in training time, similar to that of an exponential function, and increased classification rates. For classification, the softmax classifier was utilised.

\section{METHODOLOGY}

1)Xception

Two convolutional layer blocks are followed by a ReLU activation in the input flow [5].The number of filters, filter size (kernel size), and strides are all detailed in the diagram.Separable convolutional layers are also available.Max Pooling layers are also available. When there are more than one stride, the strides are mentioned as well.There are also Skip connections, where the two tensors are combined using 'ADD'.In each flow, it also shows the shape of the input tensor.

2)K-NN

Evelyn Fix and Joseph Hodges created K Nearest Neighbor in 1951.The outcome of the k-NN classification process is a class membership, which is based on the vote of neighbours.(The number $\mathrm{k}$ is a positive integer.)If $\mathrm{k}=1$, the object's closest neighbours are one.

The procedure is as follows:

1.Set $\mathrm{k}=\mathrm{n}$, where $\mathrm{n}$ refers to the number of neighbours. The number of neighbours here is one ie, $n=1$. 
2.Using a distance formula, calculate the distance between $\mathrm{k}$ neighbours. The distance formula can be euclidean, Manhattan, or other.

3. Determine the closest neighbours based on the measured distance.

4. Count data points among $\mathrm{k}$ neighbours for each class.

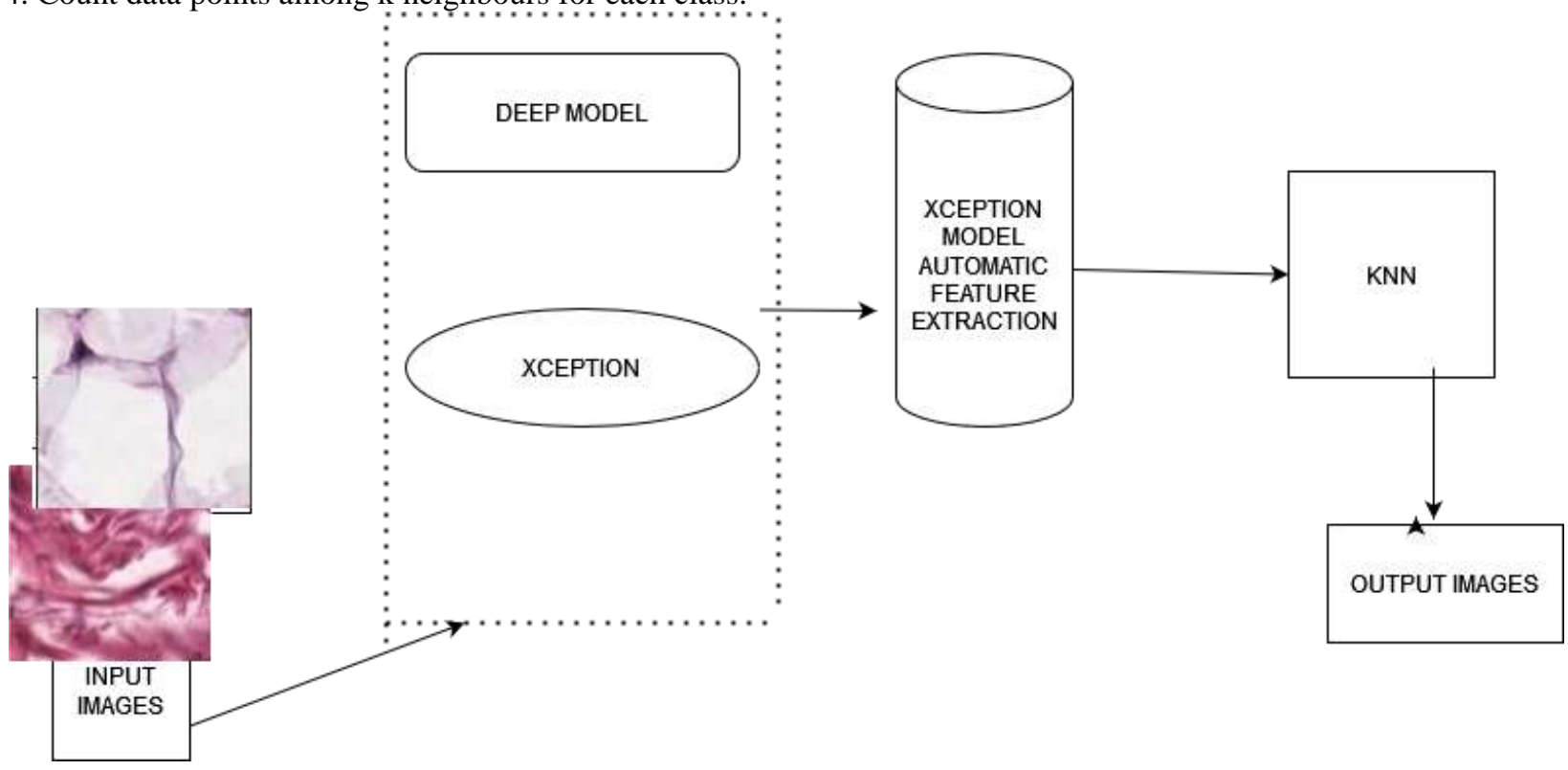

\section{RESULT}

5.create new data points based on the most neighbours.

The features are retrieved using the Xception network, and the outputs are categorised using the knn classifier with $\mathrm{k}=3$.

Evaluation metrics like precision,recall,f1-score are calculated and got an accuracy of 76 percentage.

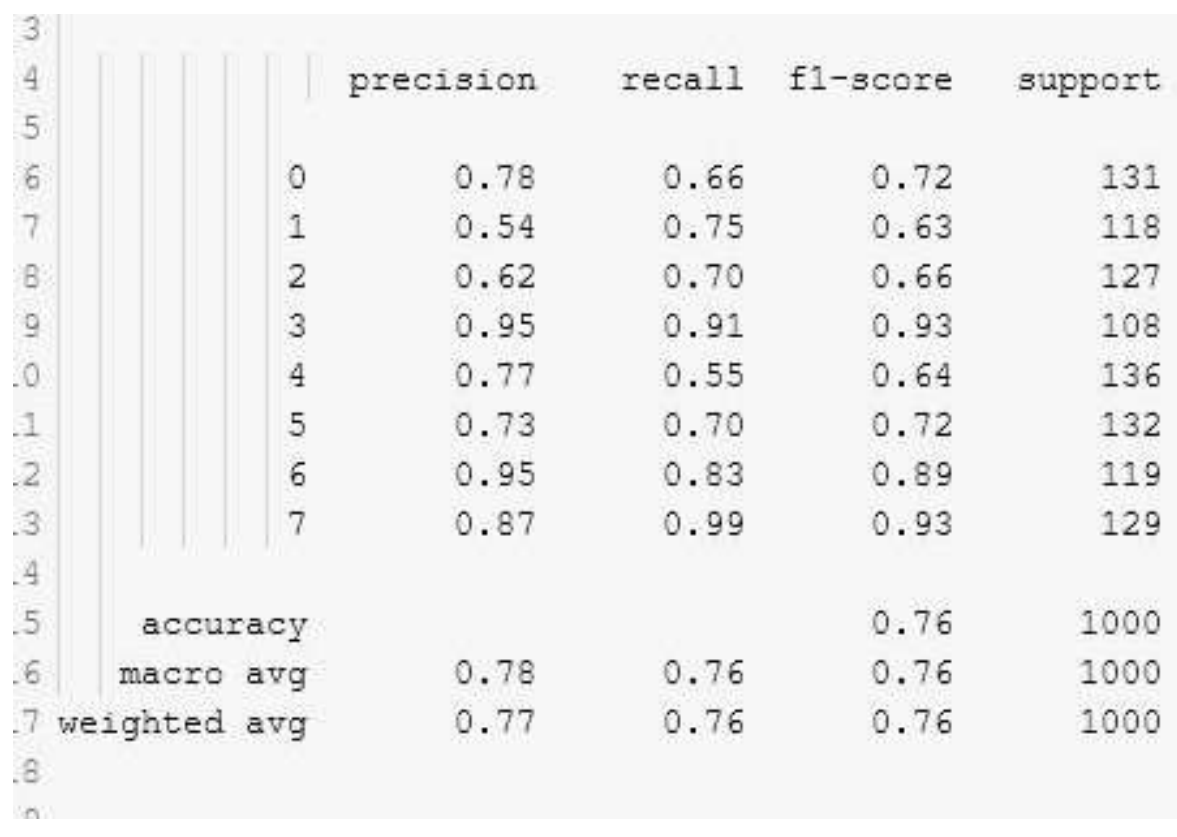




\section{CONCLUSION}

The image classification challenge was solved utilising the xception network for feature extraction and the k-nearest neighbour technique as a classifier.In the future, several hybrid techniques could be utilised for classification.

\section{REFERENCES}

1) Elene Firmeza Ohata et al. "A novel transfer learning approach for the classification of histologicalimages of colorectal cancer". In:The Journal of Supercomputing(2021), pp. 1-26.

2) Silvia Cascianelli et al. "Dimensionality reduction strategies for cnn-based classification ofhistopathological images". In:International conference on intelligent interactive multimedia systemsand services. Springer. 2018, pp. 21-30

3) Chaofeng Wang et al. "Histopathological image classification with bilinear convolutional neuralnetworks". In:2017 39th Annual International Conference of the IEEE Engineering in Medicine andBiology Society (EMBC). IEEE. 2017, pp. 4050-4053

4) Tuan D Pham. "Scaling of texture in training autoencoders for classification of histological images ofcolorectal cancer". In:International Symposium on Neural Networks. Springer. 2017, pp. 524-532.

5) Fran, cois Chollet. "Xception: Deep learning with depthwise separable convolutions". In:Proceedings ofthe IEEE conference on computer vision and pattern recognition. 2017, pp. $1251-1258$ 